\title{
AN OSCILLATORY INTEGRAL ESTIMATE ASSOCIATED TO RATIONAL PHASES
}

\author{
MAGALI FOLCH-GABAYET AND JAMES WRIGHT
}

\begin{abstract}
Let $R(t)=P(t) / Q(t)$ be a quotient of real polynomials. We show that $\int \exp (i R(t)) d t / t$ has a uniform bound with a bound depending only on the degrees of $P$ and $Q$ and not on their coefficients. Also $L^{p}$ esimates are obtained for certain associated singular integral operators.
\end{abstract}

\section{INTRODUCTION}

In [4] Stein and Wainger proved the following uniform oscillatory integral estimate:

$$
\left|p . v . \int_{-\infty}^{\infty} e^{i P(t)} \frac{d t}{t}\right| \leq C_{d}
$$

where $P$ is a real polynomial of degree $d$ and $C_{d}$ does not depend on the coefficients of $P$. As a consequence one sees that the singular integral operator

$$
H_{\gamma} f(x)=p . v . \int_{-\infty}^{\infty} f(x-\gamma(t)) \frac{d t}{t}
$$

is bounded on $L^{2}\left(\mathbf{R}^{n}\right)$. Here $\gamma(t)=\left(P_{1}(t), \ldots, P_{n}(t)\right)$ and each $P_{j}$ is a real polynomial. $L^{p}\left(\mathbf{R}^{n}\right)$ bounds for $H_{\gamma}$, when $1<p<\infty$, are also known to hold, see e.g., [5]. In this short note we extend this result to rational functions $R(t)=P(t) / Q(t)$ where $P$ and $Q$ have real coefficients. More precisely we have

\section{Theorem 1.1.}

$$
\left|p . v . \int_{-\infty}^{\infty} e^{i R(t)} \frac{d t}{t}\right| \leq A
$$

where $A$ depends only on the degrees of $P$ and $Q$ and not on their coefficients.

Remark 1.2. One thing we can do with rational functions that we cannot do with polynomials is multiply numerator and denominator by the same quantity without changing matters. For instance, we may assume that $P$ and $Q$ defining the rational function $R$ do not have a constant term. Also by multiplying the numerator and denominator by a small positive parameter $\epsilon$, we may assume that any norm of the

1991 Mathematics Subject Classification. 42B15.

The first author acknowledges financial support from CONACyT (32408-E) and DGAPAUNAM (PAPIIT IN102799). 
coefficients of $P$ and $Q$ is less than 1 . Hence if the integral in (1) is a continuous function of the coefficients of $R$, then Theorem 1.1 follows by compactness.

As a corollary to Theorem 1.1 the singular integral operator $H_{\gamma}$ is bounded on $L^{2}\left(\mathbf{R}^{n}\right)$ where $\gamma(t)=\left(R_{1}(t), \ldots, R_{n}(t)\right)$ and each $R_{j}$ is a real rational function. When $\gamma(t)=(t, R(t))$ is a plane curve, we extend the $L^{2}$ estimate to $L^{p}$ estimates.

Theorem 1.3.

$$
T f(x, y)=p \cdot v \cdot \int_{-\infty}^{\infty} f(x-t, y-R(t)) \frac{d t}{t}
$$

is bounded on $L^{p}\left(\mathbf{R}^{2}\right), 1<p<\infty$ with bounds independent of the coefficients of $R$.

In [2] it was observed that Theorem 1.1 does not extend to higher dimensions yet there are certain classes of rational phases which do extend.

In the next section we state and prove the main lemma which we need for the proofs of Theorems 1.1 and 1.3; the proof of Theorem 1.1 is then given in section 3 and the proof of Theorem 1.3 is given in the final section.

Notation: Let $A, B$ be complex-valued quantities. We use $A \lesssim B$ or $A=O(B)$ to denote the estimate $|A| \leq C_{d, d^{\prime}}|B|$ where $d$ and $d^{\prime}$ denote the degrees of $P$ and $Q$. We use $A \sim B$ to denote the estimates $A \lesssim B \lesssim A$.

\section{Preliminaries}

The key lemma in the proofs of Theorems 1.1 and 1.3 is a simple variant of a lemma in $[1]$.

Lemma 2.1. Let $P(t)=D \prod_{j=1}^{m}\left(t-t_{j}\right)=\sum_{j=0}^{m} p_{j} t^{j}$ and $Q(t)=E \prod_{k=1}^{n}\left(t-s_{k}\right)=$ $\sum_{k=0}^{n} q_{k} t^{k}$ be two nonconstant polynomials of degrees $m$ and $n$, whose roots are ordered such that $\left|t_{1}\right| \leq \ldots \leq\left|t_{m}\right|$ and $\left|s_{1}\right| \leq \ldots \leq\left|s_{n}\right|$.

Then there exists a $C=C(m, n)$ such that for any $A \geq C(m, n)$ and for any nonempty $I=\left[A\left|t_{j}\right|, A^{-1}\left|t_{j+1}\right|\right] \cap\left[A\left|s_{k}\right|, A^{-1}\left|s_{k+1}\right|\right]$,

i) $|P(t)| \sim\left|p_{j}\right||t|^{j}$ and $|Q(t)| \sim\left|q_{k}\right||t|^{k}$ for all $|t| \in I$ (hence $p_{j}, q_{k} \neq 0$ );

ii) if $j \neq k$, then for $|t| \in I$,

$$
\left|P^{\prime}(t) / P(t)-Q^{\prime}(t) / Q(t)\right| \sim 1 /|t|
$$

iii) if $j \neq k$ and $j \neq k+1$, then for $|t| \in I$,

$$
\left|(P / Q)^{\prime \prime}(t)\right| \sim|P(t) / Q(t)|\left[1 /|t|^{2}\right] .
$$


Proof Clearly for $|t| \in I$ (and any $A>1$ ),

$$
(1-1 / A)^{m}|D|\left[\prod_{\ell=j+1}^{m}\left|t_{\ell}\right|\right] \leq|P(t)| /|t|^{j} \leq(1+1 / A)^{m}|D|\left[\prod_{\ell=j+1}^{m}\left|t_{\ell}\right|\right]
$$

and similarly for $|Q(t)|$. Hence to prove i) it suffices to show

$$
|D| \prod_{\ell=j+1}^{m}\left|t_{\ell}\right| \sim\left|p_{j}\right| \quad \text { and } \quad|E| \prod_{\ell=k+1}^{n}\left|s_{\ell}\right| \sim\left|q_{k}\right| .
$$

In fact $p_{j}=(-1)^{j} D \sum_{\ell_{1}<\cdots<\ell_{m-j}} t_{\ell_{1}} \cdots t_{\ell_{m-j}}$

$$
\begin{gathered}
=(-1)^{j} D \sum_{\substack{\ell_{1}<\ldots<\ell_{m-j} \\
\ell_{1} \leq j}} t_{\ell_{1}} \cdots t_{\ell_{m-j}}+(-1)^{j} D t_{j+1} \cdots t_{m} \\
=I I
\end{gathered}
$$

and hence since $\left|t_{\ell}\right| \leq(1 / A)\left|t_{\ell^{\prime}}\right|$ whenever $\ell \leq j \leq \ell^{\prime}-1$,

$$
A|I| \lesssim|D|\left|t_{j+1}\right| \cdots\left|t_{m}\right|=|I I| .
$$

Therefore for $A>1$ large enough,

$$
\left|p_{j}\right| \sim|I I|=|D| \prod_{\ell=j+1}^{m}\left|t_{\ell}\right| .
$$

In exactly the same way we also have $\left|q_{k}\right| \sim|E| \prod_{\ell=k+1}^{n}\left|s_{\ell}\right|$ finishing the proof of i).

To prove ii) we use the formula

$$
\begin{gathered}
\frac{P^{\prime}(t)}{P(t)}-\frac{Q^{\prime}(t)}{Q(t)}=\sum_{\ell=1}^{m} \frac{1}{t-t_{\ell}}-\sum_{\ell=1}^{n} \frac{1}{t-s_{\ell}} \\
=-\sum_{\ell=j+1}^{k} \frac{1}{t-s_{\ell}}+\sum_{\ell=1}^{j} \frac{t_{\ell}-s_{\ell}}{\left(t-t_{\ell}\right)\left(t-s_{\ell}\right)}+\sum_{\ell>j} \frac{1}{t-t_{\ell}}-\sum_{\ell>k} \frac{1}{t-s_{\ell}} .
\end{gathered}
$$

Here we are assuming without loss of generality that $k>j$, and we will only concentrate on the lower bound for $\left|P^{\prime} / P-Q^{\prime} / Q\right|$ (the upper bound being much easier). For $\ell>j$ we have $\left|t-t_{\ell}\right| \geq(1-1 / A)\left|t_{\ell}\right| \geq A(1-1 / A)|t|$ and so

$$
\left|\sum_{\ell>j} \frac{1}{t-t_{\ell}}\right| \lesssim \frac{1}{A(1-1 / A)} \frac{1}{|t|} \text {. }
$$

Similarly

$$
\left|\sum_{\ell>k} \frac{1}{t-s_{\ell}}\right| \lesssim \frac{1}{A(1-1 / A)} \frac{1}{|t|}
$$

Also for $\ell \leq j,\left|t-s_{\ell}\right|,\left|t-t_{\ell}\right| \geq(1-1 / A)|t|$, and so

$$
\begin{gathered}
\left|\sum_{\ell=1}^{j} \frac{t_{\ell}-s_{\ell}}{\left(t-t_{\ell}\right)\left(t-s_{\ell}\right.}\right| \leq \frac{1}{(1-1 / A)^{2}|t|^{2}} \sum_{\ell=1}^{j}\left|t_{\ell}-s_{\ell}\right| \\
\lesssim \frac{1}{A(1-1 / A)^{2}} \frac{1}{|t|} .
\end{gathered}
$$


Finally for $t>0$,

$$
\begin{gathered}
\left|\sum_{\ell=j+1}^{k} \frac{1}{t-s_{\ell}}\right| \geq \sum_{\ell=j+1}^{k} \operatorname{Re}\left[\frac{1}{t-s_{\ell}}\right]=\sum_{\ell=j+1}^{k} \frac{t-R e s_{\ell}}{\left|t-s_{\ell}\right|^{2}} \\
\geq \frac{(1-1 / A)}{(1+1 / A)^{2}} \frac{1}{t}
\end{gathered}
$$

and similarly for $t<0$, giving us for any $|t| \in I$,

$$
\left|\sum_{\ell=j+1}^{k} \frac{1}{t-s_{\ell}}\right| \geq \frac{(1-1 / A)}{(1+1 / A)^{2}} \frac{1}{|t|} .
$$

These estimates, together with $(3)$, show

$$
\left|\frac{P^{\prime}(t)}{P(t)}-\frac{Q^{\prime}(t)}{Q(t)}\right| \gtrsim \frac{(1-1 / A)}{(1+1 / A)^{2}} \frac{1}{|t|}
$$

for all $|t| \in I$, if $A>1$ is chosen large enough, completing the proof of part ii) of the lemma.

The proof of iii) is similar to ii). Again we will only prove the harder lower bound. However now the symmetry between $P$ and $Q$ is lost and we cannot necessarily assume $j<k$. In fact in order to see explicitly why we need to impose $j \neq k+1$ as well as $j \neq k$ in this part, we will concentrate on the case $j>k+1$ (the case $j<k$ being slightly easier). We have

$$
(P / Q)^{\prime}(t)=P(t) / Q(t)\left[\frac{P^{\prime}(t)}{P(t)}-\frac{Q^{\prime}(t)}{Q(t)}\right]=P(t) / Q(t)\left[\sum_{\ell=1}^{m} \frac{1}{t-t_{\ell}}-\sum_{\ell=1}^{n} \frac{1}{t-s_{\ell}}\right]
$$

and so

$$
(P / Q)^{\prime \prime}(t)=P(t) / Q(t)\left\{\left[\sum_{\ell=1}^{m} \frac{1}{t-t_{\ell}}-\sum_{\ell=1}^{n} \frac{1}{t-s_{\ell}}\right]^{2}+\sum_{\ell=1}^{m} \frac{1}{\left(t-s_{\ell}\right)^{2}}-\sum_{\ell=1}^{n} \frac{1}{\left(t-t_{\ell}\right)^{2}}\right\} .
$$

The proof of part ii) showed

$$
\sum_{\ell=1}^{m} \frac{1}{t-t_{\ell}}-\sum_{\ell=1}^{n} \frac{1}{t-s_{\ell}}=\sum_{\ell=k+1}^{j} \frac{1}{t-t_{\ell}}+O(1 / A|t|) .
$$

Similarly

$$
\sum_{\ell=1}^{m} \frac{1}{\left(t-s_{\ell}\right)^{2}}-\sum_{\ell=1}^{n} \frac{1}{\left(t-t_{\ell}\right)^{2}}=-\sum_{\ell=k+1}^{j} \frac{1}{\left(t-t_{\ell}\right)^{2}}+O\left(1 / A|t|^{2}\right) .
$$

Therefore

$$
\begin{gathered}
(P / Q)^{\prime \prime}(t)=P(t) / Q(t)\left[\left(\sum_{\ell=k+1}^{j} \frac{1}{t-t_{\ell}}\right)^{2}-\sum_{\ell=k+1}^{j} \frac{1}{\left(t-t_{\ell}\right)^{2}}+O\left(1 / A|t|^{2}\right)\right] \\
=P(t) / Q(t)\left[2 \sum_{k+1 \leq \ell_{1}<\ell_{2} \leq j} \frac{1}{t-t_{\ell_{1}}} \frac{1}{t-t_{\ell_{2}}}+O\left(1 / A|t|^{2}\right)\right] .
\end{gathered}
$$

Note that the main term in the brackets vanishes if $j=k+1$. As in ii)

$$
\left|\sum_{k+1 \leq \ell_{1}<\ell_{2} \leq j} \frac{1}{t-t_{\ell_{1}}} \frac{1}{t-t_{\ell_{2}}}\right| \geq \sum_{k+1 \leq \ell_{1}<\ell_{2} \leq j} \operatorname{Re}\left[\frac{1}{t-t_{\ell_{1}}} \frac{1}{t-t_{\ell_{2}}}\right]
$$




$$
=\sum_{k+1 \leq \ell_{1}<\ell_{2} \leq j} \frac{t^{2}-t R e\left(t_{\ell_{1}}+t_{\ell_{2}}\right)+R e\left(t_{\ell_{1}} t_{\ell_{2}}\right)}{\left|\left(t-t_{\ell_{1}}\right)\left(t-t_{\ell_{2}}\right)\right|^{2}} \gtrsim 1 / t^{2}
$$

for $|t| \in I$, which as before completes the proof of part iii) the lemma.

Remarks 2.2. Lemma 2.1 i) shows that with respect to $P, \mathbf{R}^{+}$can be decomposed

$$
\mathbf{R}^{+}=\cup_{\ell=1}^{M} G_{\ell} \cup \cup_{\ell=1}^{M-1} D_{\ell}
$$

into disjoint intervals $(M=O(1))$ which depends on the choice of $A$ where the $D_{\ell}$ are dyadic in the sense that if $D_{\ell}=[a, b), b / a=O(1)$. On the complementary intervals $G_{\ell}$ (which we call "gaps"), if $|t| \in G_{\ell},|P(t)| \sim\left|p_{j_{\ell}}\right||t|^{j_{\ell}}$ for some $j_{\ell} \geq 0$ (and of course $p_{j_{\ell}} \neq 0$ ). See [1].

Lemma 2.1 ii) gives the bounds

$$
\left|(P / Q)^{\prime}(t)\right| \sim|P(t) / Q(t)|[1 /|t|]
$$

for $|t| \in I$ while Lemma 2.1 iii) gives analogous bounds on the second derivative of $P / Q$.

In Lemma 2.1 either $P$ or $Q$ (not both) can be allowed to be constant (various sums in the proof are then zero). However, according to the remark after the statement of Theorem 1.1, we may assume that both $P$ and $Q$ are both nonconstant from the outset.

Simple examples show that it is necessary to assume $j \neq k$ in part ii) and additionally $j \neq k+1$ in part iii) of Lemma 2.1 .

We now state a very useful estimate for one dimensional oscillatory integrals, known as van der Corput's lemma. A proof can be found in [5].

Lemma 2.3. Suppose $\phi$ is real-valued and smooth on $(a, b)$, and that $\left|\phi^{(k)}(t)\right| \geq$ $\lambda>0$ for all $t \in(a, b)$. Then

$$
\left|\int_{a}^{b} e^{i \phi(t)} d t\right| \leq C_{k} \lambda^{-1 / k}
$$

holds when either $k \geq 2$, or $k=1$ and $\phi^{\prime}(t)$ is monotonic.

\section{Proof of Theorem 1.1}

Recall that we seek to bound

$$
p . v . \int_{-\infty}^{\infty} e^{i P(t) / Q(t)} \frac{d t}{t} .
$$

We invoke Lemma 2.1 with respect to $Q(t)=\sum_{j=0}^{n} q_{j} t^{j}$ and decompose the integral into corresponding gaps and dyadic intervals according to the first remark after the 
proof of Lemma 2.1 and thus it suffices to concentrate our attention on a single gap $G$,

$$
\int_{|t| \in G} e^{i P(t) / Q(t)} \frac{d t}{t}
$$

Let us suppose $|Q(t)| \sim\left|q_{k}\right||t|^{k}$ for $|t| \in G$. We would be in trouble with estimating this integral if the derivative of $P / Q,\left(P^{\prime} Q-P Q^{\prime}\right) / Q^{2}$, behaves like $1 / t$ over a long interval. Remarkably this does not happen; Lemma 2.1 tells us that this could only occur on an interval where $|P(t)| \sim a_{k}|t|^{k}$. However on such an interval, the numerator $P^{\prime} Q-P Q^{\prime}$ possesses some cancellation. To see this, set $D(t)=$ $q_{k} P(t)-p_{k} Q(t)=\sum d_{\ell} t^{\ell}$. Note that $d_{k}=0$. We now invoke Lemma 2.1 again but now with respect to $D$ and split $\mathbf{R}^{+}$into gaps and dyadic intervals adapted to $D$. Hence we may reduce ourselves to bounding

$$
e^{i p_{k} / q_{k}} \int_{|t| \in G \cap H} e^{i\left[P(t) / Q(t)-p_{k} / q_{k}\right]} d t / t=e^{i p_{k} / q_{k}} \int_{|t| \in G \cap H} e^{i D(t) / q_{k} Q(t)} d t / t
$$

where $H$ is a gap for $D$, say $|D(t)| \sim\left|d_{j}\right||t|^{j}$ for $|t| \in H$. According to Lemma 2.1 i), $j \neq k$ since $d_{k}=0$. Note that

$$
\frac{d}{d t}\left(\frac{D}{q_{k} Q}\right)=\frac{D}{q_{k} Q}\left(\frac{D^{\prime}}{D}-\frac{Q^{\prime}}{Q}\right)
$$

and so by ii) of Lemma 2.1 we see that

$$
\left|\frac{d}{d t}\left(\frac{D}{q_{k} Q}\right)(t)\right| \gtrsim\left(\left|d_{j}\right| / q_{k}^{2}\right)|t|^{j-k-1}
$$

on $G \cap H$ since $k \neq j$. We consider the two cases $k>j$ and $k<j$. When $k \leq j-1$ we split the integral

$$
\begin{gathered}
\int_{|t| \in G \cap H} e^{i D(t) / q_{k} Q(t)} \frac{d t}{t}=\int_{\substack{|t| \in G \cap H \\
|t| \geq \Theta}} e^{i D(t) / q_{k} Q(t)} \frac{d t}{t}+\int_{\substack{|t| \in G \cap H \\
|t| \leq \Theta}} e^{i D(t) / q_{k} Q(t)} \frac{d t}{t} \\
I+I I
\end{gathered}
$$

where $\Theta$ satisfies $q_{k}^{2} /\left|d_{j}\right|=\Theta^{j-k}$ (recall that $q_{k} \neq 0$ and $\left.d_{j} \neq 0\right)$.

By van der Corput's lemma we see that $I=O(1)$ (since we are using Lemma 2.3 with $k=1$, we need to first split the integration in $I$ into $O(1)$ intervals where the derivative of the phase is monotone). For $I I$ we estimate

$$
\begin{gathered}
|I I|=\left|\int_{\substack{|t| \in G \cap H \\
|t| \leq \Theta}}\left(e^{i D(t) / q_{k} Q(t)}-1\right) \frac{d t}{t}\right| \\
\lesssim \frac{1}{\left|q_{k}\right|} \int_{\substack{|t| \in G \cap H \\
|t| \leq \Theta}}|D(t)| /|Q(t)| d t / t \lesssim\left|d_{j}\right| / q_{k}^{2} \int_{|t| \leq \Theta}|t|^{j-k-1} d t=O(1),
\end{gathered}
$$

which completes the estimate when $k \leq j-1$. The case $k \geq j+1$ is similar and this finishes the proof of Theorem 1.1. 


\section{Proof of Theorem 1.3}

Here we proceed in a similar fashion as in the proof of Theorem 1.1, first decomposing $\mathbf{R}^{+}$into gaps and dyadic intervals with respect to $Q$, reducing ourselves to bounding the operator

$$
T_{G} f(x, y)=\int_{|t| \in G} f(x-t, y-P(t) / Q(t)) \frac{d t}{t}
$$

where $|Q(t)| \sim\left|q_{k}\right||t|^{k}$ for $|t|$ in the gap $G$. We will need to estimate the second derivative of $P / Q$ and so (see part iii) of Lemma 2.1) we need to do more than subtract $p_{k} / q_{k}$ from $P / Q$, i.e., conjugating $T_{G}$ with a translation. Conjugating $T_{G}$ with the invertible linear transformation $L(x, y)=(x, x-C y)$ for a suitable $C \neq 0$ followed by a translation allows us to subtract from $P(t) / Q(t)$ any linear polynomial $\alpha+\beta t, \beta \neq 0$, without affecting the $L^{p}$ operator norms of $T_{G}$. The idea is to choose $\alpha$ and $\beta$ appropriately so that in the difference

$$
P(t) / Q(t)-\alpha-\beta t=(P(t)-[\alpha+\beta t] Q(t)) / Q(t),
$$

the numerator $N(t)=P(t)-[\alpha+\beta t] Q(t)=\sum_{j} n_{j} t^{j}$ has vanishing $k t h$ and $(k+1) t h$ coefficients, i.e., $n_{k}=n_{k+1}=0$, putting us in a position to use part iii) of Lemma 2.1. A little linear algebra shows that this can be achieved if and only if $q_{k}^{2} \neq$ $q_{k-1} q_{k+1}$.

We will now see that this is indeed the case since $G$ is a gap, i.e., the $k t h$ root $s_{k}$ of $Q$ is separated from the $(k+1) t h$ root $s_{k+1}$. Recall that $G$ is of the form $\left[A\left|s_{k}\right|, 1 / A\left|s_{k+1}\right|\right]$ and the $k t h$ coefficient $q_{k}$ of $Q$ is related to the roots of $Q$ by the bounds in (2), $\left|q_{k}\right| \sim|E| \prod_{\ell=k+1}^{n}\left|s_{\ell}\right|$; hence

$$
q_{k}^{2} \sim E^{2} \prod_{\ell=k+1}^{n}\left|s_{\ell}\right|^{2}
$$

For $q_{k-1} q_{k+1}$ we have

$$
\begin{aligned}
q_{k-1} q_{k+1}= & E^{2}\left[\sum_{\ell_{1}<\ldots<\ell_{n-k+1}} s_{\ell_{1}} \cdots s_{\ell_{n-k+1}}\right]\left[\sum_{\ell_{1}<\ldots<\ell_{n-k-1}} s_{\ell_{1}} \cdots s_{\ell_{n-k-1}}\right] \\
= & E^{2} \sum_{\substack{\ell_{1}<\ldots<\ell_{n-k+1} \\
\ell_{1}^{\prime}<\ldots<\ell_{n-k-1}^{\prime}}} s_{\ell_{1}} \cdots s_{\ell_{n-k+1}} s_{\ell_{1}^{\prime}} \cdots s_{\ell_{n-k-1}^{\prime}} .
\end{aligned}
$$

Since in each summand defining $q_{k-1},\left|s_{\ell_{1}}\right| \leq\left|s_{k}\right| \leq\left(1 / A^{2}\right)\left|s_{k+1}\right|$, we have

$$
\left|s_{\ell_{1}} \cdots s_{\ell_{n-k+1}} s_{\ell_{1}^{\prime}} \cdots s_{\ell_{n-k-1}^{\prime}}\right| \leq \frac{1}{A^{2}} \prod_{\ell=k+1}^{n}\left|s_{\ell}\right|^{2} .
$$

Therefore by (5)

$$
\left|q_{k-1} q_{k+1}\right| \lesssim \frac{1}{A^{2}} E^{2} \prod_{\ell=k+1}^{n}\left|s_{\ell}\right|^{2}=O\left(\frac{1}{A^{2}} q_{k}^{2}\right)
$$

and we see that $q_{k}^{2} \neq q_{k-1} q_{k+1}$ if $A$ is chosen large enough. 
Hence we can choose $\alpha$ and $\beta$ such that the $k t h$ and $(k+1) t h$ coefficients $n_{k}, n_{k+1}$ of $N(t)=P(t)-(\alpha+\beta t) Q(t)$ vanish. We note here that if the resulting $\beta$ is zero, then translation by $\alpha=p_{k} / q_{k}$ alone gives $n_{k}=n_{k+1}=0$. As noted above it suffices for us to prove $L^{p}$ bounds for

$$
S_{G} f(x, y)=\int_{|t| \in G} f(x-t, y-N(t) / Q(t)) \frac{d t}{t} .
$$

We decompose $\mathbf{R}^{+}$further into gaps and dyadic intervals adapted to $N$ reducing ourselves to estimating the operator

$$
S_{G, H} f(x, y)=\int_{|t| \in G \cap H} f(x-t, y-N(t) / Q(t)) \frac{d t}{t}
$$

where $|N(t)| \sim\left|n_{j}\right||t|^{j}$ for $|t| \in H$ say. Also $j \neq k$ and $j \neq k+1$ since $n_{k}=n_{k+1}=0$. By conjugating $S_{G, H}$ with a dilation we may assume that $q_{k}=n_{j}=1$. We will use Theorem 5.1 in [3] to see that $S_{G, H}$ has uniform $L^{p}$ bounds with $1<p<\infty$.

In fact, using the notation in [3], $S_{G, H}$ is a convolution operator with convolution kernel $K=\sum_{\ell \in Z} \mu_{\ell}^{(\ell)}$ where $\mu_{\ell}^{(\ell)}$ is the $(x, y) \rightarrow\left(2^{\ell} x, 2^{\ell(j-k)} y\right)$ dilate of the measure $\mu^{(\ell)}$ defined by

$$
\mu^{(\ell)}(\phi)=\int_{|s| \in 2^{-\ell}(G \cap H) \cap[1,2]} \phi\left(s, R_{\ell}(s)\right) \frac{d s}{s} .
$$

Here $R_{\ell}(s)=2^{\ell(k-j)} N\left(2^{\ell} s\right) / Q\left(2^{\ell} s\right)$ and since $R_{\ell}(s)=O(1)$ by Lemma 2.1 part i), the family of measures $\mu^{(\ell)}$ is uniformly supported in a fixed compact set. In this context, Theorem 5.1 in [3] takes the following form:

Proposition 4.1. Suppose that the uniform decay estimate

$$
\left|\widehat{\mu}^{(\ell)}(\zeta)\right| \leq C|\zeta|^{-\epsilon}
$$

holds for some $C, \epsilon>0$ independent of $\ell$. Then $f \rightarrow f * K$ is bounded on $L^{p}\left(\mathbf{R}^{2}\right), 1<$ $p<\infty$ with bounds depending only on $p, \epsilon$ and $C$.

We remark that it is important that $k \neq j$ in the dilations $(x, y) \rightarrow\left(2^{\ell} x, 2^{\ell(j-k)} y\right)$ defining $K$; otherwise, the application of Theorem 5.1 in [3] would require the additional cancellation $\widehat{\mu}^{\ell}(0, \eta) \equiv 0$ which is simply not the case here.

Thus it suffices to verify (6) with $C$ independent of $\ell$ and the coefficients of the rational function $R$. In fact we will show

$$
\left|\widehat{\mu}^{(\ell)}(\zeta)\right| \lesssim|\zeta|^{-1 / 4}
$$

where

$$
\widehat{\mu}^{(\ell)}(\zeta)=\int_{|s| \in I} e^{i\left[\xi s+\eta R_{\ell}(s)\right]} \frac{d s}{s}
$$

and $I=2^{-\ell}(G \cap H) \cap[1,2]$.

Lemma 2.1, part iii) implies that $\left|R_{\ell}^{\prime \prime}(s)\right| \gtrsim 1$ for $|s| \in I$ (recall that $q_{k}=n_{j}=1$ ). Thus van der Corput's lemma shows that (7) holds whenever $|\xi| \leq|\eta|^{2}$. On the 
other hand, when $|\eta|^{2} \leq|\xi|$, we can write $e^{i \xi s}=1 / i \xi(d / d s)\left(e^{i \xi s}\right)$ and integrate by parts, using the fact $R_{\ell}^{\prime}(s)=O(1)$ (Lemma 2.1, part ii) ) to see that in this case,

$$
\left|\widehat{\mu}^{(\ell)}(\zeta)\right| \lesssim|\eta| /|\xi| \lesssim|\zeta|^{-1 / 2}
$$

establishing (7).

This completes the proof of Theorem 1.3.

\section{REFERENCES}

[1] A. Carbery, F. Ricci, J. Wright, Maximal functions and Hilbert transforms associated to polynomials, Revista Mat. Ibero. 14 (1998), 117-144.

[2] M. Folch-Gabayet, J. Wright, An estimation of a family of singular integrals, to appear in Studia Math.

[3] F. Ricci, E.M. Stein, Multiparameter singular integrals and maximal functions, Ann. Inst. Fourier (Grenoble) 42 (1992), 637-670.

[4] E.M. Stein, S. Wainger, The estimation of an integral arising in multiplier transformations, Studia Math. 35 (1970), 101-104.

[5] E.M. Stein, Harmonic Analysis, Princeton Univ. Press (1993).

Instituto de Matemáticas, UnAM, Area de la Investigación Científica, Circuito ExteRior, Ciudad Universitaria, MÉxico, D.F. 04510

E-mail address: folchgab@matem.unam.mx

School of Mathematics, University of Edinburgh, JCMB, King's Buildings, Mayfield RoAd, Edinburgh EH9 3JZ, SCOtLAND

E-mail address: wright@maths.ed.ac.uk 